By F. Croxon Deller, M.D., M.R.C.P.

(Temporary Honorary Physician, Metropolitan Hospital, Acton Hospital, Honorary Consulting Physician, Wilson Hospital, Mitcham.).

The clinical picture of the catarrhal child is one which is unfortunately too well known to need minute description, although careful consideration of the disease process is an essential if there is to be any advance in our therapeutic efforts to relieve or cure the child of its symptoms: Up to date, therapy has been disjointed, haphazard and not too successful although it is agreed by many clinicians that the child may be lucky enough to grow out of the catarrhal state as puberty approaches. But many of these children carry their catarrhal condition with them all through life. No follow-up records, however, have been made of those children who apparently recover spontaneously and it is believed, based on very careful adult history taking, that there is a great tendency for them to return to their catarrhal state later in adult life, even after a period of apparent quiesence of upwards of fifteen years. Be that as it may, it does not alter the significant fact that the rational therapeutic approach to the catarrhal child is at present inadequate. Expectorants; with or without belladonna, sedative linctuses, tonsillectomy, adequate or excessive dosage of vitamins, change of air, open-air schools, courses of sulpha drugs, or of vaccines or desensitisation therapy, ultra violet light - these and many other treatments are used with no real relationship the one to the other. Or worse, no therapeutic effort at all.

\section{Natural History of the Catarrhal State}

Often one or other parent or a member of the household is catarrhal or suffers with bronchitis and this immediately suggests that an infective factor is most important from the aetiological viewpoint. The catarrhal state is always heralded by an initial attack of acute bronchitis, associated with either teething or measles or whooping cough or as part of a pneumonic process. From this initial attack the child never fully recovers. The cough persists, not often associated with any visible sputum since most often it is swallowed. Each succeeding cold seems to "go on to the chest" and make the cough more evident. The catarrhal condition is one which affects the whole of the respiratory epithelium and nasal obstruction and catarrh are usual concomitants. The adenoids enlarge, the child becomes a mouth breather and the tonsils become oedematous and swollen. Persistent mild pyrexia up to $99 \cdot 4^{\circ}$ or bouts of pyrexia, swelling of the cervical and tonsillar [sic] glands, anorexia or capricious appetite all follow in the $\stackrel{c}{.}$ wake of this catarrhal picture. Inevitably as the disease process continues, the child fails to increase $\overrightarrow{0}$ in weight and becomes undernourished, although it usually develops mentally in a normal manner. Thus by the time the child has reached the age of eight or nine years the typical description is one of bodily under-development with an intelligent quotient far in advance of its apparent age.

The virulence or frequency of succeeding attacks of respiratory infection is the keystone of future life for the child. If mild, the catarrhal state is 8 little save an inconvenience and it is in this type of case that the prognosis is good and the "usual i treatments" effective. But if severe, the mor- i bidity rate is considerable. Recurrent attacks of $\underset{ \pm}{ \pm}$ "bronchitis" with slowly progressing deformity of $\vec{\sim}$ the chest and eventually the development of $\vec{\omega}$ emphysema and of asthma. The relationship of $\frac{\circ}{7}$ bronchitis and asthma has been fully discussed elsewhere (Deller, I946). To reiterate, however, asthma has been shown to be a symptom of respiratory catarrh in most cases and to be due to exces- $\overrightarrow{0}$ sive vagal stimulation caused by tenacious secretiog or sputum covering an inflamed and swolle respiratory mucous membrane. Moncrieff (I93) described the inter-relationship of cough and bronchial spasm to bronchitis, but did not carry the argument to the conclusions made possible by bronchoscopic investigations.

That other symptoms follow this pageant only becomes apparent with careful history taking and successful therapy. Vomiting is a common accompaniment occurring at night or in the morning and is due to coughing, although the parent rarely associates the two conditions. Attacks of diarrhoea or looseness of the bowel are not uncommon. Generalised adenopathy, a toxic look, toxic skin eruptions often including erythema nodosum, listlessness and headache are common. In brief, the whole picture becomes one of a slowly progressive intoxication. The experimental work about to be $\frac{7}{2}$ described will show that this toxaemia comes from a low-grade generalised respiratory infection. N

\section{Experimental Investigation}

Careful interrogation of the child and of the parents was the first step and the results attained from this are shown above in the review of the natural history of the disease.

The clinical examination was next carried out

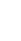


diligently, the general findings of which have already been described. Auscultation revealed a variable amount of information-the classical description of a cough with no physical signs being true unless the breath sounds immediately after coughing were auscultated-when soft râles could be heard especially at the bases of the lungs. At the other end of the scale, the signs of emphysema were present together with multitudinous râles and rhonchi of all types.

The upper respiratory tract was most carefully examined in all cases. The nasal airway was found to be inefficient usually. The nasal mucous membrane was swollen, oedematous and often covered with secretion or scabs. The inferior turbinate bones were usually swollen and transillumination of the antra showed loss or absence of translucency. Pus was often seen lying in the floor of the nares. Post-nasal discharge was a common finding and the adenoids were found to be enlarged in nearly all cases. The posterior pharyngeal wall was often studded with swollen areas of oedematous lymphoid tissue and the tonsils, if present, were usually swollen and oedematous, but rarely showed any infection within the follicles. The tonsillar [sic] and cervical glands were usually enlarged and shotty.

Two interesting points were noted in relationship to the tonsils. Firstly from careful history taking it was found that the removal of both tonsils and adenoids had usually produced a deterioration in the child's condition over a period of two years, often precipitating asthma: and secondly, that the child rarely complained of any soreness of the throat or difficulty in swallowing such as is usual in the subacute follicular tonsillitis of adults; further reference to these points will be made later.

From these simple clinical findings two deductions were possible: first, that the infection was a generalised catarrhal condition involving the whole of the respiratory epithelium: second that the persistence of the condition, the gradual deterioration of the child and general clinical picture was due to the fact that the infection was subacute and rarely completely quiescent.

To prove this latter concept bronchoscopic investigation of these children was carried out and the findings here described are based on a study of more than fifty such cases. The mucous membrane of the pharynx, epiglottis, larynx, trachea and bronchi was found to be oedematous and often hyperaemic. The whole respiratory mucous membrane, especially that of the trachea, was covered by secretion: sometimes colourless and tenacious, at other times thick and purulent: with all gradations between. In the trachea and main bronchi there were present always small swollen patches, not unlike the lymphoid follicles on the posterior pharyngeal wall already mentioned and in somet cases definite ulceration was noted. Swabs were taken from the tracheal and bronchial mucous membrane and cultures grew a mixed collection of pathogenic organisms with the haemolytic strepto:cocci of viridans type predominating. These find $\overrightarrow{\bar{F}}$ ings coupled with the appearances of the uppef respiratory tract confirmed the concept that the catarrhal child suffers from a generalised respiram tory epithelial infection and that no part of the respiratory tract is immune from the infective process.

\section{Therapeutic Applications}

As a corollary to these findings it is obvious that a planned therapeutic approach must be made fo every catarrhal child and that the ubiquitous bottle of medicine cannot by itself affect a cure. The respiratory mucous membrane from the nares tN the smallest bronchioles must be considered as a. whole and not in separate compartments.

The upper respiratory tract needs first attentiono The antra need especial attention first as it is use less to remove the tonsils and adenoids unless the antra are free from infection. The tonsils, unles badly scarred or obviously infected, should be left alone. This conclusion was reached on simple clinical experience alone. Later the work of Isoo Griffiths (I937) was rediscovered, and to tô. reference should be made as it appears to be of grea importance in connection with the catarrhal childo He showed that the tonsil has a protective influence् over the nasopharynx and conversely that the lymphatic drainage of the antra, accessory nasaf sinuses and adenoids passed down beneath the tonsil to the submaxillary gland, the cervical chaip and even the tracheo-bronchial gland, and it is te. be noted that it is these glands which enlarge in the catarrhal child. The protective influence of the tonsil explains the reason why tonsillectomy ofter precipitates asthma since such removal allows further infection of the respiratory mucous memê brane to occur.

The treatment of upper respiratory catarrh therefore, appears without doubt to be antrab wash-outs, followed by the instillation of $15,00 \underline{\underline{\alpha}}$. units of penicillin into each antrum and adenoidro ectomy, with the encouragement of nasal breathing, if need be by means of a specially made dent stent worn at night, to help the normal cilial action of the epithelium.

The bronchoscope provides the other therapeutio weapon. Bronchial suction with aspirators of suif able size and bronchial lavage, using a $2 \%$ sodiurn bicarbonate solution or hydrogen peroxide ro volso per cent, until all secretion is removed is mos important: once this has been adequately per 
formed the bronchial mucous membrane is sprayed with a solution of $\mathrm{I}$ : Iooo propamidine, using either Io-20 c.c. of the solution in all or a solution of proflavine hemisulphate I: I000 can be used.

Inhalation of penicillin through some form of nebuliser is a most useful adjunct to therapy and will usually clear up any residual infection following on the above therapeutic programme. Inhalation of the penicillin spray should be through the mouth: exhalation through the nose will clear any remaining nasal catarrh. The most useful solution is:-

$\begin{array}{lr}\text { Penicillin } & \text { roo,000 units } \\ \text { Glycerine } & 2 \cdot 5 \text { c.c. } \\ \text { Water } & \text { ad. Io c.c. }\end{array}$

The glycerine sweetens the taste and provides a better nebule. A child can usually use upwards of 3 c.c. a day of this solution in a Pag or Maw's inhaler.

Following this therapy, simple tonic measures, adequate vitamin intake, especially of ascorbic acid, general hygienic measures and breathing exercises are necessary until recovery is complete.

The youngest child on whom this whole programme has been performed so far was aged four years and six months. At present it is only the technical difficulties of sprays and aspirators that preclude its use in younger children.

\section{Case Reports}

These six cases are selected from over fifty cases as each illustrates certain points or symptoms of importance.

Case I. S.H. aet ro: This child had been subject to repeated attacks of bronchitis since aged $2 \frac{1}{2}$ years and had never been free of a cough which was unproductive, persistent and resistent to all forms of treatment. When first seen she had a temperature of $102 \cdot 4^{\circ}$ with a severe unproductive cough, dyspnoea and some degree of bronchial spasm. It was also noted at this time that pus was present in both nostrils, lying under the inferior turbinate bones. Once the acute attack had subsided it was found that the catarrhal signs in the chest persisted and on investigation both antra were found to be completely opaque, both clinically and radiologically and an X-ray of the chest showed a catarrhal condition, but no other abnormality.

Under general anaesthetic both antra were punctured and thick pus welled up from each cannula. The antra were washed out and I0,000 units of penicillin introduced into each. No other therapeutic measures were attempted.
The child made an immediate recovery and sinceo that time there has been no cough at all. Both antra have completely cleared; both transillumi-z

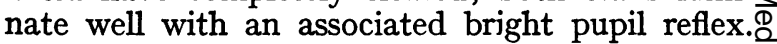
No signs of bronchitis or bronchial spasm have. been discovered even on repeated examinations $\overrightarrow{\vec{F}}$ during the past fifteen months.

The child has put on weight, eats well and has? developed normally.

This case illustrates the fundamental fact that $\frac{\vec{n}}{\overline{0}}$ much of the so-called lower respiratory catarrh is entirely secondary to an infection in the upper\% respiratory tract to which treatment must be $\vec{\circ}$ directed.

Case II. W.S. aet 9: Advice was sought in this $\vec{\omega}$ case because of sudden attacks of diarrhoea. On clinical examination it was found that the childo was under weight. That there was marked nasal obstruction and much post-nasal discharge whichN was very purulent. No other abnormality wasi discovered save for some bronchial catarrh. It. was considered that the enteritis was secondary ton the respiratory catarrh.

At operation, both antra contained pus and the 9 adenoids were found to be enlarged. The antra $\vec{\longrightarrow}$ were washed out and the adenoids removed. Since the operation all signs of respiratory catarrh have cleared up and no further attacks of diarrhoea hagee occurred during the past twelve months.

This case illustrates one of the associated sym toms of respiratory catarrh.

Case III. S.D. aet I2: This child had had asthma for six years. Careful questioning showed that the initial attack had come on within three days of the $\frac{\bar{\Phi}}{2}$ development of acute bronchitis and that the con- $\Rightarrow$ dition had persisted ever since. She had had a? very severe attack eighteen months ago and this was followed by an illness that was diagnosed as anterior poliomyelitis, but which was not apparently typical and might well have been a peri- 3 pheral neuritis. (Two other cases with this complication have been seen.)

On examination the child was found to be thin, 8 the cervical glands were enlarged, the tonsils $₹$ swollen and oedematous, nasal obstruction was $ᄋ$ present and the antra did not transilluminate well. There were scattered râles and rhonchi to be heard all over the chest.

The child was introspective and in order to $\widetilde{N}$ eliminate any purely psychological factor, she was $N$ given an anaesthetic and narcosis therapy was tried. This produced no change in her condition. Two months later under a further anaesthetic the antra were washed out, the adenoids removed and bronchial lavage and suction performed. She re- $\stackrel{\Phi}{+}$ mained perfectly well for a period of four months; then she developed an attack of bronchitis which, $\overline{0}$ however, responded dramatically to inhalations of 
penicillin. She has remained well since. There has been no attack of asthma since the bronchial lavage.

This case illustrates the general catarrhal picture and the noticeable improvement which followed the line of therapy described. The avoidance of a further infection of the respiratory tract does need considerable care and thought, but early treatment with inhalations of penicillin may prove to be the correct answer to this problem.

Case IV. I.W. aet II : This child had asthma since he was two years old and he presented the usual clinical picture. In this case there have been repeated bouts of pyrexia for no apparent cause during the past four years.

At operation bronchial lavage and suction only was performed. Although at the time he had been quiescent for a period of two months, much pus was aspirated from the bronchial tree. He made an uninterrupted recovery and has had no further asthma over twelve months. The cough entirely disappeared for six months and then returned, when it was noticed there was some upper respiratory catarrh.

Inhalation of penicillin via the mouth with exhalation through the nose completely cleared this in a matter of two weeks. He has since remained symptom free.

Case V. S.M. aet II: This child was apparently a typical allergic case of asthma and hay fever, although desensitisation had produced no improvement at all.

At operation pus was found in both antra, the adenoids were enlarged and in the bronchial tree much pus was aspirated although the child was in a quiescent stage at the time of operation.

This child has put on weight and looks very much healthier, the appetite has improved and all symptoms referable to the respiratory tract have disappeared. This has been maintained over a period of six months.

Case VI. A.C. aet 8: This child was brought on account of asthma which she had developed when five years old. Careful questioning, however, revealed that she had had attacks of bronchitis following measles when two and a half years old and that the asthma developed after a very severe recrudescence of the catarrhal state.

On examination the child was thin and there were typical expiratory rhonchi to be heard all over the chest. The upper respiratory mucous membrane was swollen and infected and there was a deficient nasal airway due to swelling of the inferior turbinate bones. There was lack of translucency of the antra and tonsils which were definitely infected.

In this case it was decided that the upper respiratory tract should be dealt with first. The antra were washed out and pus was found in small $\stackrel{\circ}{0}$ quantities in each. Penicillin was then instilled. The tonsils and adenoids were removed. This helped her general condition somewhat, but she $\underset{\infty}{\mathbf{\infty}}$ had no real improvement in the asthmatic state.

Three months later a second anaesthetic was given for the bronchial lavage and suction. It was noted by the anaesthetist that this second anaes- -0 thetic was considerably less difficult to administer 듬 than the previous one.

At bronchoscopy much secretion was seen in the $\frac{\vec{\phi}}{8}$ bronchial tree and the whole of the bronchial mucous membrane was swollen and there were ${ }^{\circ}$ small ulcers to be seen scattered over the tract. $\overrightarrow{ }$

The child has improved very much since this $\overrightarrow{-}$ second treatment. Her weight has increased and ${ }_{\stackrel{\omega}{\omega}}$ her appetite is considerably better. She still had 0 slight attacks of asthma during the night for the 3 first month, but after a short course of inhalation therapy, using a Collison inhaler containing a mixture of penicillin and sodium sulphacetamide, this $\stackrel{ \pm}{+}$ cleared dramatically and she has now remained $\overrightarrow{-}$ symptom free for over six months.

This case has been reported in order to demon- $\bigcirc$ strate that it is necessary to deal with both the upper and the lower respiratory tracts in severe 3 cases. In this case the clearing up of the infection in the upper respiratory tract did not lead to the $\overrightarrow{0}$ elimination of the lower respiratory infection and furthermore it is to be noted that in severe cases. it may be necessary to repeat bronchial lavage are suction on more than one occasion, or to follow the first treatment with inhalation therapy.

Cases have been tried with inhalation therapy

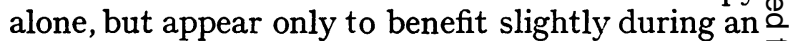
acute attack of bronchitis.

Therefore every case needs individual attention and a planned therapeutic approach.

\section{Results of Treatment}

All these cases had first been treated on the "usual lines" and had not responded at all satisfactorily. Any case which did show a beneficialo response to such therapy was excluded from the series.

Twenty cases have been watched for a period of $\triangle$ twelve months or more. Of these, sixteen have remained symptomatically cured and of the fourN cases which relapsed further therapy removed the 0 symptoms rapidly. All the cases have improved ${ }^{N}$ very considerably in general health. Of the thirty $\omega^{2}$ cases watched for less than a year, it appears that? there is likely to be a similar percentage of success.co

With increasing experience and experiment the therapeutic programme should be simplified and? improved upon, but it is felt that bronchial suction, $\frac{0}{0}$ especially in the cases associated with asthmatic 
attacks is the most important feature of the therapy. The relationship of upper respiratory tract infection to the catarrhal state must be emphasised, but this does not constitute the whole picture which is that of a generalised respiratory catarrh.

\section{Summary}

I. The pathogenesis and clinical features of the catarrhal child have been fully discussed.

2. The bronchoscopic findings in these children have been described.

3. The rôle of upper respiratory tract infection in the catarrhal child has been stressed.

4. A new therapeutic programme for the catarrhal child has been suggested.
5. The results of this new therapy, with reports on six illustrative cases, have been given.

\section{REFERENCES}

I. DELLER, F. C. (1946), Post-grad. med. J., 22, 43.

2. MONCRIEFF, A. (I934), "Diseases of Children," Garrod, Batten, Thursfield and Paterson, London, p. 4I3.

3. GRIFFITHS, IVOR' (1937), Lancet, ii, 723.

The great encouragement and help both academic $\frac{\bar{\sigma}}{\overline{0}}$ and practical which I have received from Mr. J. D. $\frac{\pi}{\widetilde{T}}$ McLaggan, F.R.C.S., and Mr. Ivor Griffiths, $\stackrel{\mathbb{Q}}{ }$ M.S., F.R.C.S., must not be overlooked, for without them much of the understanding of the catarrhal $\vec{\circ}$ child which I believe I have now, would have been:lost to me. Much has been learned also from my $\vec{\omega}$ anaesthetic colleagues, especially Dr. G. Hochs-s child and Dr. Woodfield Davies who have so oftenoo borne with me very patiently.

\title{
RADIOTHERAPY AND CANCER
}

\author{
By D. W. Smithers, M.D., D.M.R.
}

(Director of the Radiotherapy Department of the Royal Cancer Hospital (Free) and the Royal Free Hospital; Radiotherapist Brompton Hospital for Diseases of the Chest, and the West End Hospital for Nervous Diseases. $)^{2}$

\section{Introduction}

Fifty years ago in November, I895, Wilhelm Conrad Röntgen discovered X-rays; within one year Henri Becquerel had discovered radioactivity, and within three years Marie and Pierre Curie had isolated radium. Röntgen, Becquerel, and the Curies opened up new fields for research and their discoveries have had the most profound influence on the progress of science. One direct outcome of their work was the introduction to medicine of a valuable new method of treatment, but there is probably no type of treatment that has such a wide application and has been used for so long which is so little understood or its value so little appreciated by the general body of medical men.

Radiotherapy is the use of certain types of ionising radiations in the attack upon disease. Its most important, though by no means only, use in medicine is in the cure and the relief of suffering due to the scourge of cancer. A scourge because, although it is a curable disease in its early stages, it is responsible for the death of more adults than any other condition, except heart disease, and because it is second to none in its capacity to produce fear and suffering.

\section{(I) The Physical Basis of Radiotherapy}

Radiation is a process of transferring energy from one place to another; radiotherapy applies this energy to living tissues which are abnormal either in structure or in function. In canger treatment it is employed to destroy maligna cells in such a way as to give the natural defen of the body the maximum aid possible in dealing with the disease and in repairing the damage done

The types of radiation in general use at the present time are $\mathrm{X}$-rays, produced at voltages ranging from about 50,000 to several hundred thousand volts, and the gamma rays and beta rays from radium. Beta rays are particles (electrons) and $\mathrm{X}$ and gamma rays are waves. These waves: however, behave very like particles in that their energy is concentrated in units (photons), the $e^{3}$. energy in each unit depending on the wavelength of the radiation-the shorter the wavelength the greater the energy of the photon.

To understand the application of these various types of radiation in medicine it is necessary to study the underlying physical principles involved These radiations affect matter, both organic and. inorganic, in fundamentally the same way. Thes produce changes in the atoms of the substances they irradiate, either by knocking electrons righe out of the atoms (ionisation) or, by displacin electrons within the atoms (excitation). In either case the properties of the affected atom are change and this change may initiate a whole series of chemical reactions. With inorganic materials the results of the irradiation may be slight, such as change of colour of the material, but with biologicaf materials the changes produced may be consider 